\title{
Um Programa de Intervenção para Transtorno de Ansiedade Social com o Uso da Realidade Virtual
}

\author{
Yhann Hafael Trad Perandré , 2, * $^{2}$ \\ Orcid.org/0000-0002-4483-9612 \\ Verônica Bender Haydu ${ }^{2}$ \\ Orcid.org/0000-0002-4522-8338
}

${ }^{1}$ Universidade Federal do Paraná, Jandaia do Sul, PR, Brasil

${ }^{2}$ Universidade Estadual de Londrina, Londrina, PR, Brasil

\section{Resumo}

A Realidade Virtual (RV) foi utilizada nesse estudo como recurso terapêutico em uma intervenção analítico-comportamental para dois participantes com transtorno de ansiedade social. Os objetivos foram avaliar os efeitos terapêuticos do programa de intervenção e o simulador quanto à capacidade de gerar senso de presença e respostas de ansiedade. O programa consistiu em: (a) sessão inicial, (b) linha de base, (c) intervenção com exposição à RV, (d) encerramento e (e) follow up (1 e 3 meses após a intervenção). O senso de presença, ansiedade e respostas galvânicas da pele foram registrados em cada sessão com exposição e inventários de ansiedade, depressão e fobia social ao término de cada fase. Foram formuladas análises funcionais baseadas na ocorrência de comportamentos apresentados em contexto social no intervalo entre as sessões. O simulador produziu ansiedade e níveis elevados de presença ao longo das exposições; e ambos participantes apresentaram redução dos níveis de ansiedade ao fim do programa e generalização para o contexto natural. Conclui-se que a intervenção com exposição à RV apresentou efeito terapêutico e promoveu repertório de enfrentamento frente a situações de interação social.

Palavras-chave: Realidade virtual, transtorno de ansiedade social, terapia analítico-comportamental, senso de presença, resposta galvânica da pele.

\section{A Treatment Program for Social Anxiety Disorder by Using Virtual Reality}

\begin{abstract}
Virtual Reality (VR) was used in this study as a therapeutic tool in a behavior-analytic intervention with two subjects who had social anxiety disorder. The goals were to assess the therapeutic effects of the intervention program and the VR simulator with regard to the ability to generate sense of presence and anxiety responses. The program consisted of: (a) initial session, (b) baseline, (c) intervention sessions

* Endereço para correspondência: Universidade Federal do Paraná (UFPR) - Campus Jandaia do Sul, Rua Doutor João Maximiano, 426, Vila Operária, Jandaia do Sul, PR, Brasil 86900-000. Fone: (41) 3208-6190. E-mail: yhann.perandre@hotmail.com

Verônica Bender Haydu é bolsista Produtividade em Pesquisa da Fundação Araucária.
\end{abstract}


with exposure to VR, (d) closing session, (e) follow up (up to one and three months after treatment). Sense of presence, anxiety and galvanic skin response were reported in each exposure therapy session and anxiety, depression and social phobia inventories were reported at the end of each stage. Functional analyses were formulated based on behaviors occurring in social contexts between sessions. The simulator produced anxiety and high levels of presence during exposure; and both participants had reduced levels of anxiety at the end of intervention and generalization to the natural context. In conclusion, behavior-analytic intervention with exposure to VR was effective and fostered a repertoire for coping with situations of social interaction.

Keywords: Virtual reality, social anxiety disorder, behavior-analytic therapy, sense of presence, galvanic skin response.

\section{Un Programa de Intervención para la Trastorno de Ansiedad Social con el Uso de la Realidad Virtual}

\section{Resumen}

La realidad virtual (RV) fue empleada en este estudio como herramienta terapéutica en un programa de intervención analítico-conductual para dos participantes con trastorno de ansiedad social. Los objetivos fueron evaluar los efectos terapéuticos del programa de intervención y el simulador en cuanto a la capacidad de generar presencia y respuestas de ansiedades. El programa consistió en: (a) sesión inicial, (b) línea base, (c) intervención con exposición a la RV, (d) finalización y (e) seguimiento (1 y 3 meses después de la intervención). La sensación de presencia, ansiedad y la respuesta galvánica de la pele fueron registrados en cada sesión con exposición y inventario de la ansiedad, depresión y fobia social al final de cada fase. Análisis funcionales fueron formulados en base a la ocurrencia de las conductas de interacción social en el intervalo entre las sesiones. El simulador produce ansiedad y niveles elevados de presencia a lo largo de las exposiciones y ambos participantes presentaron reducción de los niveles de ansiedad al final de lo programa, con generalización de los resultados para un contexto natural. Se concluye que la intervención analítico-conductual con la exposición a la RV tuvo efecto terapéutico y aprendieron un repertorio de afrontamiento de esas situaciones.

Palabras clave: Realidad virtual, trastorno de ansiedad social, terapia de la conducta, sensación de presencia, respuesta galvánica de la piel.

Novas tecnologias têm sido cada vez mais aceitas e incorporadas como recurso útil na área da Psicologia tanto no meio científico quanto na prestação de serviços, como a clínica psicológica. A utilização de recursos tecnológicos pode contribuir para agilidade do processo terapêutico e para o desenvolvimento de formas cada vez mais criativas e produtivas de ação (Barbosa, 2013). Como recurso complementar à psicoterapia, a tecnologia de Realidade Virtual (RV) possibilita atuação dinâmica e ativa tanto do terapeuta quanto do cliente e abre espaço para investigações sistemáticas sobre a eficácia de programas de intervenção terapêuticos que incluem essa tecnologia.
Os estudos sobre terapia com o uso de RV começaram a ganhar força especialmente nas últimas duas décadas (e.g., Klinger et al., 2005; Price, Mehta, Tone, \& Anderson, 2011; Slater, Pertaub, Barker, \& Clark, 2006; Wallach, Safir, \& Bar-Zvi, 2011). Nesses estudos, a RV surgiu como um recurso facilitador da técnica de exposição; como um ambiente controlado para imersão de indivíduos com transtorno de ansiedade social (fobia social); e, possibilita a modelagem de comportamentos sociais, por meio de contingências programadas (e.g., Harris, Kemmerling, \& North, 2002; Morina, Brinkman, Hartanto, \& Emmelkamp, 2014; Price et al., 2011). Assim, a RV passou a ser considerada uma nova e pro- 
missora ferramenta que pode ser usada em intervenções psicológicas. Entretanto, questões são levantadas quanto a: (a) efetividade da RV como recurso terapêutico quando comparada a outras formas de tratamento (e.g., Kampmann et al., 2016; Klinger et al., 2005), (b) validade de programas de intervenção que utilizam a RV como recurso principal (e.g., Roy et al., 2003), e (c) ao modo como as fobias específicas podem ser avaliadas por meio de biofeedback (e.g., Wiederhold, Jang, Kim, \& Wiederhold, 2002) ou por meio de autorrelatos, inventários e questionários (e.g., Harris et al., 2002).

Um dos aspectos a ser considerado nas intervenções com a RV consiste na capacidade de o simulador promover senso de presença, que pode ser definido como "o sentimento de 'estar lá' no ambiente virtual", o qual envolve respostas públicas e/ou privadas evocadas por estímulos discriminativos e mantidas por consequências que o ambiente produz (comportamentos operantes) e respostas (públicas e/ou privadas) eliciadas por estímulos do ambiente virtual (comportamentos respondentes) (Zacarin et al., 2017, p. 259). Senso de presença é especialmente relevante em técnicas terapêuticas como a exposição com prevenção de respostas e a dessensibilização sistemática, visto que para a utilização da RV nessas técnicas é necessário que o simulador de RV apresente um conjunto de situações que provoquem desconforto ou ansiedade. De acordo com Qu, Brinkman, Ling, Wiggers e Heynderickx (2014), a exposição a essas situações pode ser programada para ocorrer de modo gradual, aumentando paulatinamente o nível de aversão, conforme a tolerância do indivíduo.

A exposição gradual aos estímulos temidos pode ser feita após um reconhecimento adequado do fenômeno tratado. Em um estudo proposto por Holt, Heimberg, Hope e Liebowitz (1992), o transtorno de ansiedade social foi analisado a partir da ansiedade sentida em diferentes contextos. Os autores propuseram 24 situações distintas que poderiam ser resumidas em quatro grupos situacionais comuns: (a) interação verbal e social formal, (b) interação verbal e social informal, (c) ser observado, (d) assertividade. Os grupos situacionais propostos por Holt et al. subsidiaram estudos experimentais sobre ansiedade social e o conceito foi reapresentado por Klinger et al. (2005) e Roy et al. (2003) como: (a) ansiedade de desempenho (dificuldade em desempenhar atividades em contexto social, como falar em público), (b) ansiedade de intimidade (incapacidade do indivíduo iniciar conversações, e de criar e manter vínculos afetivos), (c) ansiedade de observação (dificuldade em executar atividades sob observação de outras pessoas, como operar um caixa eletrônico ou falar ao telefone em um shopping center cheio), (d) ansiedade de assertividade (dificuldade em expressar e afirmar sua opinião e posicionamento em relação a algo e proteger seus interesses). Em um estudo mais recente desenvolvido por Heijden e Brinkman (2011), a RV foi utilizada baseada no conceito de ansiedade de observação, no qual algumas pessoas discursavam por alguns minutos para uma audiência virtual e eram avaliados por meio da qualidade da interação verbal que apresentavam. A caracterização do transtorno baseada em grupos situacionais enfatiza os contextos nos quais o indivíduo pode apresentar repertório comportamental insuficiente de enfrentamento das situações de interação social.

Outra caracterização do transtorno de ansiedade social é promovida enquanto processo comportamental e enfatiza a descrição das variáveis mantenedoras do transtorno a partir da análise funcional dos comportamentos apresentados pelo indivíduo (Leonardi, Borges, \& Cassas, 2012). Essa análise funcional como análise comportamental descritiva da categoria diagnóstica do transtorno de ansiedade social é feita a partir da operacionalização dos seguintes aspectos: (a) das classes de resposta em níveis molares, (b) das consequências, (c) dos eventos antecedentes à resposta, (d) da história de reforço, e (e) do tratamento derivado dessa avaliação funcional (Sturmey, 1996; Silvares \& Meyer, 2000). Em um estudo relatado por Rocha, Bolsoni-Silva e Verdu (2012), foi destacado que indivíduos com transtorno de ansiedade social podem apresentar déficits em habilidades sociais em um contexto situacional específico e não em outro, evidenciando a relevância da análise funcional. 
Em uma análise sobre os processos comportamentais da ansiedade social, Zamignani e Banaco (2005) identificaram que os indivíduos podem apresentar certos comportamentos como taquicardia, alteração da resposta galvânica da pele, hiperventilação, sensações de afogamento ou sufocamento, sudorese, dores e tremores diante de estímulos do ambiente que adquiriram propriedades eliciadoras a partir de processos de condicionamento respondente (por exemplo, sons e ruídos característicos de grandes aglomerações). Ao mesmo tempo, o indivíduo pode apresentar comportamentos operantes mantidos por reforço negativo, como a fuga, que interrompem a apresentação desses estímulos (por exemplo, o uso de fones de ouvidos para atravessar grandes centros urbanos) ou a esquiva diante de estímulos que antecedem a estimulação aversiva (por exemplo, optar por horários alternativos para desempenhar suas atividades cotidianas). Em alguns casos, a esquiva bem sucedida pode ser mantida por reforço positivo, por exemplo, permissão para não comparecer em reuniões importantes etc. Portanto, comportamentos respondente e operante se inter-relacionam numa análise completa do transtorno de ansiedade social.

Os objetivos da terapia comportamental na intervenção do transtorno de ansiedade social consistem em reduzir respostas reflexas, suprimir respostas de fuga e esquiva, desenvolver relações sociais mais reforçadoras e habilidades sociais, e ensinar a manejar ou enfrentar problemas (Beidel et al., 2014; Nobile, Garcia, \& Bolsoni-Silva, 2017; Sampaio \& Bueno, 2011; Stevens, Peters, Abraham, \& Hermann, 2014). Sampaio e Bueno (2011) apresentaram uma intervenção para medo de falar em público (transtorno de ansiedade social específico) que consistiu em arranjar contingências de reforço para que o participante se expusesse a audiências não punitivas e, gradualmente, o nível de aversividade da audiência era aumentado. Tal procedimento visou o fortalecimento de respostas de enfrentamento e o enfraquecimento das respostas de esquiva. Ao final da intervenção, a participante do estudo foi capaz de realizar uma apresentação oral que lhe garantiu titulação acadêmica. Segundo os autores, o resultado do estudo em nível satisfatório só foi possível devido às estratégias aplicadas, que incluíam controle ambiental aliado ao estabelecimento de uma hierarquia dos eventos geradores de ansiedade.

A falta de controle do ambiente natural (in vivo) pode tornar a exposição muito aversiva, levando a uma resposta de esquiva da própria condição terapêutica (Anderson et al., 2013). Os programas de intervenção que utilizam a RV como recurso terapêutico, por sua vez, permitem o controle de algumas contingências de interação entre o usuário e o avatar (personagem simulado), úteis em programas de intervenção para tratamento de transtorno de ansiedade social. Um estudo recente, realizado por Roy et al. (2003), comparou a tecnologia da RV com terapias para transtorno de ansiedade social. Um grupo experimental (G1) exposto a um protocolo de intervenção de 12 sessões de RV foi comparado a outro grupo experimental (G2), exposto a intervenção baseada em Cognitive Behavior Therapy (CBT) e a um terceiro grupo (G3), que não recebeu a intervenção (lista de espera). Nesse experimento, os três grupos foram avaliados no pós-teste e ficou evidente a redução dos escores médios, obtidos por meio da aplicação da Liebowitz Social Anxiety Scale aos grupos experimentais (G1 e G2) em relação ao G3, sem diferença estatística significante entre o G1 e o G2. Resultados semelhantes foram verificados em outros estudos que também compararam a efetividade da RV com a CBT no tratamento para transtorno de ansiedade social (e.g., Klinger et al., 2005; Wallach, et al., 2011). Esses estudos demonstraram que se pode observar efeito terapêutico em intervenções com o uso de RV, assim como, em intervenções por outras terapias, como a CBT. No entanto, resultados diferentes foram obtidos por Kampmann et al. (2016) em um estudo clínico randomizado controlado, resumido a seguir.

Kampmann et al. (2016) compararam a eficácia de uma Terapia de Exposição à Realidade Virtual (Virtual Reality Exposure Therapy VRET) com uma Terapia de Exposição in vivo (in vivo Exposure Therapy - iVET). Sessenta participantes com diagnóstico de transtorno de ansiedade social foram distribuídos randomicamente em três grupos: dois de tratamento e um 
controle (lista de espera). A VRET era feita em cenários que possibilitavam interação verbal com seres humanos virtuais (avatares) e a iVET era feita em situações não-virtuais (real-life) semelhantes às virtuais, sendo ambos os procedimentos individualizados. Ao se comparar os resultados dos grupos que receberam tratamento, verificou-se que ambos demonstraram, na comparação das avaliações realizadas antes e depois das intervenções, diminuição da frequência dos comportamentos de esquiva, aumento na duração das interações verbais e redução do estresse percebido, quando comparados com a lista de espera. Os participantes que receberam iVET, mas não os que receberam VRET, melhoraram com relação ao medo de avaliação negativa, ao desempenho no falar, e apresentaram redução da ansiedade geral e da depressão em relação àqueles em lista de espera. Os autores concluíram que a VRET pode reduzir as queixas de transtorno de ansiedade social generalizada e que "futuras melhorias tecnológicas e das interações sociais virtuais psicológicas podem aumentar ainda mais a eficácia da VRET em casos de transtorno de ansiedade social" (p. 147).

Os estudos descritos anteriormente demonstraram que os tratamentos com o uso da RV podem ser efetivos. Os resultados foram obtidos por meio de testes, inventários e questionários de autorrelato, além das medidas fisiológicas, como a taxa de batimento cardíaco e a condutância elétrica da pele. Um estudo realizado por Wiederhold et al. (2002) avaliou o uso de medidas fisiológicas como indicador de melhora clínica em participantes, que apresentavam medo de voar, submetidos a um procedimento com o uso da RV. Foram monitoradas a frequência cardíaca, a resposta galvânica da pele e a temperatura corporal de 22 participantes não fóbicos $\mathrm{e}$ 36 participantes fóbicos ao longo de seis sessões de exposição à RV. Os autores relataram diferença significativa entre as medidas da resposta galvânica da pele entre os participantes fóbicos e não fóbicos durante as exposições à RV. As medidas fisiológicas voltaram aos níveis de linha de base para os participantes não fóbicos, enquanto que permaneceram por mais tempo em níveis elevados para os participantes fóbicos. Ao final da intervenção, 33 dos 36 participantes fóbicos apresentaram melhora clínica (taxa de sucesso de 91,6\%). Entretanto, a avaliação da resposta galvânica foi feita a partir da média dos grupos. Uma medida mais fidedigna poderia consistir em uma avaliação dos dados apresentados pelo indivíduo se comparados com dados dele mesmo, ao longo das sessões.

Com base nas investigações acerca do uso da tecnologia de RV aplicada ao contexto das intervenções clínicas (Barbosa, 2013; Kampmann et al. 2016; Morina et al., 2014; Wallach et al., 2011; Wiederhold et al., 2002), o presente estudo teve como objetivos: (a) avaliar os efeitos de um programa de intervenção analítico-comportamental com o uso da RV para tratamento de indivíduos com transtorno de ansiedade social; (b) avaliar um simulador de RV projetado para transtorno de ansiedade social quanto à capacidade de gerar senso de presença e respostas de ansiedade registradas por medida de biofeedback da resposta galvânica da pele e pela $S u b$ jective Units of Distress Scale - SUDS (Wolpe, 1973/1978).

\section{Método}

\section{Participantes}

Participaram do estudo dois estudantes universitários, ambos do sexo masculino, com 20 e 27 anos, P1 e P2, respectivamente. Os participantes foram selecionados a partir de uma entrevista com roteiro semiestruturado e com a aplicação dos testes BDI, BAI e SPIN, com o objetivo de verificar se: (a) atendiam ao diagnóstico psicológico de transtorno de ansiedade social, baseado nos critérios do DSM-5 (American Psychiatric Association [APA], 2013); (b) não apresentavam hipótese diagnóstica de depressão ou comorbidade; (c) não estavam em tratamento psicológico ou farmacológico concomitante ao estudo; e (d) dispunham de tempo para comparecer às sessões até duas vezes por semana, por aproximadamente uma hora. Os participantes assinaram o Termo de Consentimento Livre e Esclarecido, aprovado por Comitê de Ética em Pesquisa.

O Participante 1 (P1) tinha 20 anos, solteiro (namorava), era estudante universitário, morava 
com os irmãos desde os 17 anos para estudar. Até então, morava com os pais em uma cidade pequena do interior. Relatou pouca intimidade com os pais e irmãos. Ele relatou que os percebia como "autoridades, em maior posição hierárquica". Diante de qualquer interação verbal com eles, evitava contato visual e como consequência as interações eram pontuais e breves. Em decorrência, era interpretado como "bravo, nervoso" e como subproduto emocional sentia-se triste. O mesmo padrão repetia-se com professores, demais familiares, instrutor de música (fez aula de teclado e canto). Sentia "medo de colegas de sala, sobretudo os mais importantes e populares". P1 relatou medo de falar em seminários e as apresentações acadêmicas e artísticas eram precedidas por muita ansiedade. Assim como nas apresentações, toda interação verbal era marcada por taquicardia, perda de voz, tremor, medo, freezing, sentia-se inseguro, relatava baixa autoestima e fugia das situações.

P2 tinha 27 anos, solteiro (namorava), estudante universitário, morava em casa compartilhada com outras duas pessoas, com as quais não mantinha contato íntimo. Anteriormente, residia com a mãe na capital do Estado. Relatou ter cursado o Ensino Médio em 3 anos a mais do que o previsto, mudou-se de cidade e escolas por muitas vezes ao longo da sua formação e sentia especial dificuldade de interação social nesses ambientes. Sentia muito desconforto em entrar e permanecer em salas de aula, devido ao fato da iminência da interação com os colegas de sala, precisar falar sobre si e sobre assuntos cotidianos. Diante desse tipo de interação, sentia tremor, rubor, pernas inquietas, ansiedade. Já realizou psicoterapia em vários períodos desde os 16 anos de idade, sendo a última vez já há alguns anos, com o objetivo de tratar o transtorno de ansiedade social, sem êxito. Além disso, sentia dificuldade em ir e permanecer em restaurantes, manipular talheres e comer em frente a outras pessoas. P2 apresentava repertório comportamental restrito, relatava viver uma "rotina": diariamente ia para a universidade, mas diante das salas de aula dirigia-se para outro lugar, como o calçadão da universidade, bibliotecas ou outro lugar com baixa probabilidade de interação com os colegas de sala. A baixa frequência às aulas produzia subprodutos emocionais como "sentimento de culpa e raiva".

\section{Local e Materiais}

As sessões foram realizadas em uma sala da Clínica Psicológica de uma Universidade pública. A sala dispunha de duas cadeiras e uma mesa para apoio do material e equipamento necessário às sessões.

Foi utilizado o aparelho de biofeedback Mindifield ${ }^{\circledR}$ eSense Skin Response para monitorar a resposta galvânica da pele (ver https:// goo.gl/kCidld) e o simulador de RV Vitua.Therapy ${ }^{\circledR}$ - composto por um Oculus $\operatorname{Rift}{ }^{\circledR}$, dois computadores laptops, um joystick Razer ${ }^{\circledR}$ Hydra com dois controles, e fones de ouvido externos. O simulador apresentava cenários específicos projetados para o tratamento de transtorno de ansiedade social. Os cenários exibidos apresentavam contexto para possíveis interações sociais do participante com os avatares e possível desempenho de tarefas pré-determinadas. Os cenários foram projetados em uma sequência de eventos, mas sem prejuízo caso fossem apresentadas cenas em ordem alternativa. A primeira cena consistia em uma praça de alimentação de um shopping center e nenhum avatar compunha o cenário; a partir da segunda cena eram introduzidos avatares gradualmente. A segunda cena consistia em um caixa eletrônico possível de ser operado, localizado na praça de alimentação; a terceira cena acontecia em uma das lanchonetes da praça de alimentação; a quarta e quinta cenas eram executadas em uma parte da mesma praça de alimentação, que continha mesas e cadeiras.

\section{Instrumentos}

Os instrumentos especificados a seguir foram usados para mensuração dos comportamentos. A Escala de Unidades Subjetivas de Desconforto (Subjective Units of Distress Scale - SUDS; Wolpe 1973/1978) indicava o nível de ansiedade e desconforto sentido durante a exposição à RV em um escore que variava entre 0 e 10 . Escores mais próximos de zero indicavam nenhum nível de ansiedade e escores mais próximos de 10 indicavam maiores níveis de ansiedade. 
O Inventário de Senso de Presença (ISP) consistia em 14 itens que descreviam a exposição à RV em termos de estímulos virtuais, estímulos do ambiente não virtual, reações fisiológicas durante a exposição e comportamentos apresentados ao longo da exposição. As respostas eram dadas em escala Likert de 0 (discordo totalmente) a 4 (concordo totalmente). $\mathrm{O}$ escore total apresentava o nível em que o participante se sentia presente no ambiente virtual

O Inventário de Ansiedade de Beck (Beck Anxiety Inventory - BAI; Cunha, 2001) avaliava a frequência da emissão de comportamentos compatíveis com o transtorno de ansiedade. O BAI era composto por 21 itens em escala Likert de 0 a 3 . Um escore total de 0 a 10 correspondia ao nível Mínimo; de 11 a 19, Leve; de 20 a 30, Moderado; de 31 a 63, Grave.

O Inventário de Depressão de Beck (Beck Depression Inventory - BDI; Cunha, 2001) avaliava a frequência da emissão de comportamentos compatíveis com a depressão. O BDI era composto por 21 itens em escala Likert de 0 a 3 . Um escore total de 0 a 11 correspondia ao nível Mínimo; de 12 a 19, Leve; de 20 a 35, Moderado; de 36 a 63, Grave.

O Inventário de Fobia Social (Social Phobia Inventory - SPIN; Osório, Crippa, \& Loureiro, 2009) avaliava a frequência da emissão de comportamentos compatíveis com a fobia social. O SPIN era composto por 17 itens em escala Likert de 0 a 4 e o escore total acima de 19 indicava a presença desses sintomas.

A Folha de Registro Comportamental consistia em uma folha na qual o participante relatava as situações de interação social que havia enfrentado ou esquivado, no intervalo entre as sessões, a fim de proceder as análises funcionais e verificar a generalização da intervenção para outros contextos.

Além desses instrumentos, foi usada a Folha de Instrução da exposição à RV, que: (a) apresentava uma breve explicação dos comportamentos envolvidos nas relações interpessoais que produziam o medo e/ou esquiva das situações, (b) subsidiava a compreensão das relações comportamentais proporcionadas pela exposição à RV, e (c) descrevia os objetivos das exposições à $R V$.

\section{Procedimento}

O programa era composto por uma sessão inicial; cinco sessões de linha de base; oito sessões de intervenção com exposição à $\mathrm{RV}$; uma sessão de encerramento; duas sessões de follow up, sendo uma após 1 mês do encerramento e outra após 3 meses do encerramento. A quantidade de sessões para cada fase do procedimento proposta servia como referência e estava sensível às necessidades de cada participante. Para ambos os participantes, a sessão inicial e a sessão de encerramento foram realizadas em duas etapas, visto que eram realizadas também a aplicação dos testes e inventários.

Sessão Inicial. A sessão inicial consistia em entrevista com roteiro semiestruturado, seguida pela apresentação do estudo, dos objetivos e dos procedimentos. A participação era registrada com a assinatura no Termo de Consentimento Livre e Esclarecido e, somente então, eram aplicados os testes BDI, BAI e SPIN.

Fase de Linha de Base. A linha de base foi realizada em cinco sessões. Os sensores do aparelho de biofeedback eram colocados nos dedos do participante e o aparelho era ligado por 40 $\mathrm{s}$ antes do início da utilização do Virtua.Therapy. O participante era exposto em todas as sessões de linha de base à Cena 1 , um cenário que não requeria interações sociais, com o objetivo de ensinar ao participante como utilizar o equipamento e os acessórios, como os joysticks, a função de "pegar objetos", movimentação no cenário e o head-tracking. A instrução dada ao participante era

explore o cenário e suas áreas, pelo tempo que achar necessário para aprender lidar com o equipamento e familiarizar-se com a tecnologia. Nos primeiros momentos, evite movimentos bruscos da cabeça, pois poderá sentir algum mal-estar, como vertigem.

A exposição era programada para ocorrer por 5 min e poderia ser encerrada antes, a pedido do participante.

Após o término da exposição, o aparelho de biofeedback permanecia ligado por mais 40 s para que houvesse estabilização dos dados. $\mathrm{O}$ participante respondia a SUDS e o ISP imediatamente após a exposição à RV, e em seguida a 
uma avaliação da experiência com o simulador e com os acessórios. Era perguntado ao participante "O que você sentiu?", "Como você agiu para lidar com isso?", "Há algo que você acredita ser importante comentar?". O mesmo procedimento ocorria em todas as sessões de linha de base.

Fase de Intervenção. Durante a fase de intervenção, além da exposição à $\mathrm{RV}$, outros recursos terapêuticos eram introduzidos: a respiração diafragmática, a análise funcional de comportamentos com o uso da Folha de Registro. Esses recursos compunham o programa de intervenção. A respiração diafragmática era ensinada ao participante, durante a primeira sessão da fase de intervenção, consistindo em explicar como utilizar o músculo diafragmático durante a respiração, facilitando a entrada de ar pelos pulmões e maior oxigenação com menor custo. Para isso, era instruído:

concentre-se em sua respiração, procure sentir os movimentos de inspirar e expirar, procure deixá-los cada vez mais lentos, vá sentindo o ar entrar pela sua boca, depois saindo, preste atenção em sua barriga, que se enche quando você inspira, puxando o ar para dentro e vai murchando quando você solta o ar para fora, expirando. Procure notar como é relaxante prestar atenção em sua respiração, e o quanto você tem controle sobre ela, sinta todo seu corpo contribuir para a respiração, relaxando, concentre-se nessa sensação de prazer.

O procedimento de intervenção com a exposição à RV consistia em: (a) rapport; (b) análise funcional com a Folha de Registro Comportamental; (c) respiração diafragmática; (d) exposição à RV; (e) aplicação da SUDS e do ISP; e (f) a avaliação da experiência com o simulador e com os acessórios. Assim como na fase de linha de base, o aparelho de biofeedback era ligado 40 $\mathrm{s}$ antes da exposição à $\mathrm{RV}$ e desligado $40 \mathrm{~s}$ após o término da exposição.

Os cenários da exposição simulavam interações sociais e o desempenho de atividades em contexto social simulado. As cenas eram divididas em exposições que pudessem produzir ansiedade de observação (Cena 2), ansiedade de assertividade (Cena 3), ansiedade de desempe- nho (Cena 4) e ansiedade de intimidade (Cena 5). A ordem dos cenários foi ajustada com base nos relatos da sessão inicial, isto é, conforme o grau de dificuldade de enfrentar as situações. A mesma sequência foi utilizada para ambos os participantes. A qualquer momento a exposição à $\mathrm{RV}$ poderia ser interrompida pelo participante ou experimentador, caso o participante apresentasse sinais de mal-estar (por exemplo, náuseas, dores de cabeça, vertigem), caso a exposição fosse mais aversiva do que o participante pudesse suportar ou por outro motivo apresentado pelo participante. No entanto, interrupções não foram necessárias ao longo das intervenções.

Após o desligamento do aparelho de biofeedback, o participante respondia a SUDS e o ISP e, então, era feita a avaliação da experiência da exposição à RV. Essa avaliação consistia em uma investigação oral, com perguntas do seguinte tipo: "Como você se sentiu ao longo da exposição?" e "O que você fez depois e como você se sentiu por isso?". O objetivo dessa avaliação era sumarizar, ao encerramento da sessão, os comportamentos apresentados pelo participante durante a sessão, as condições antecedentes e consequentes, ou seja, estruturar possíveis análises funcionais no contexto da exposição e durante a sessão.

Sessão de Encerramento. Durante a sessão de encerramento eram aplicados os testes pós-intervenção: BDI, BAI e SPIN.

Sessões de Follow Up. Foram realizadas 1 mês e 3 meses após a última sessão com a RV. Nessas sessões, o participante foi exposto novamente aos cenários de RV, precedido de exercício de respiração diafragmática e com o monitoramento da resposta galvânica da pele. No final, o participante respondia novamente ao BDI, BAI e SPIN.

\section{Resultados}

Durante a primeira sessão de intervenção foi apresentada a Folha de Registro Comportamental e os participantes foram instruídos a preenchê-la entre uma sessão e outra, a fim de subsidiar a formulação de análises funcionais junto ao participante nas sessões seguintes. A 
partir da segunda sessão de intervenção até a última sessão de follow up, P1 apresentou nove Folhas de Registro preenchidas com, respectivamente: 4, 4, 1, 2, 3, 4, 2, 4 e 4 episódios comportamentais cada. O P2 entregou duas folhas preenchidas com um episódio comportamental descrito em cada.

Os episódios comportamentais relatados por P1, por meio das Folhas de Registro, foram, em maior parte, em contexto de intimidade e em desempenho de atividades em contexto social. As situações que P1 relatou em maior frequência foram apresentações de trabalho acadêmico, interações verbais com colegas de sala e familiares, e interações verbais sobre sua orientação sexual. Nos episódios descritos nas Folhas 1, 2 e 3, que descreviam conversas com amigos sobre orientação sexual, observou-se como antecedentes: rodas de conversa e caminhadas pelo calçadão da universidade. P1 relatou que nessas ocasiões, apesar de ter pensado em expor aos amigos sobre sua orientação sexual, não o fez. Relatou haver direcionado a conversa para temas alheios à questão da sua sexualidade. As consequências observadas foram: (a) o alívio por não ter se exposto para os colegas (função de esquiva); (b) sentimento de baixa autoestima, evidenciado pelo relato: "fiquei mal por não conseguir responder corretamente [às perguntas sobre sua orientação sexual] e por ficar nervoso".

Nos episódios comportamentais que descreviam situações em ambiente acadêmico, como perguntas aos professores, apresentações de trabalhos e seminários, P1 relatou diferentes classes de respostas ao longo do processo de intervenção. Quanto às perguntas em sala de aula, P1 descreveu nas primeiras folhas que somente realizava as perguntas nos momentos em que os demais colegas conversavam entre si. Como consequência, observou-se o alívio em evitar a interação com os demais presentes na situação (esquiva). Entretanto, esse padrão comportamental produzia prejuízos ao $\mathrm{P} 1$ quando não havia a possibilidade de realizar perguntas na ausência da observação de outras pessoas. Ao final do período de intervenção, as descrições das ocasiões em que P1 interagia com o professor, apresentava também presença dos demais colegas. P1 relatava sentir-se feliz por conseguir expor-se e frequentemente era respondido adequadamente pelo professor (consequência reforçadora para o comportamento de fazer perguntas em sala de aula).

Outras descrições, nas Folhas 4, 6 e 7, apresentavam situações de interação social com pessoas da família. Diante das situações de interação, P1 fez o enfrentamento e relatou na Folha 4: "fiquei contente por conseguir conversar e não ficar quieto apenas"; Folha 6: "a conversa terminou bem. Ele [tio] ficou feliz por eu ter falado um pouco inclusive sobre o filho dele"; Folha 7: "eles [primos e irmãos] me olhavam rindo $e$ fiquei meio sem graça. Parei de rir, mas apesar disso foi legal". Observou-se um aumento na frequência do comportamento de interação social com os familiares após o início da intervenção, em relação à frequência relatada na entrevista inicial e observou-se que os comportamentos apresentados eram reforçados na própria situação de interação com os familiares.

O P2 apresentou pouco engajamento na tarefa de responder às Folhas de Registro. Na Folha 1, P2 relatou um encontro com amigos da namorada em um ônibus. P2 descreveu não ter interagido de acordo com o contexto, apenas verbalizou quando chamado, sentiu-se "envergonhado" e relatou "não passei uma boa imagem para as amigas dela [namorada]". P2 sentiu-se "constrangido" por apresentar pouca habilidade social. Na Folha 2, P2 descreveu que diante da necessidade em obter informações sobre o curso que fazia, dirigiu-se à secretaria. Apresentou taquicardia e sudorese, realizou as perguntas e não obteve respostas satisfatórias. Sentiu-se "frustrado e chateado" por não conseguir enfrentar adequadamente a situação. De modo geral, P2 apresentava repertório comportamental restrito e insuficiente para interações sociais ao início da intervenção e, observou-se a partir de relatos verbais, que ao decorrer das sessões de intervenção P2 apresentava repertório comportamental mais adaptativo. Próximo ao final do procedimento, P2 relatou que passou a frequentar eventos sociais, em que interagiu verbalmente com outros convidados e com os amigos da namorada.

Os resultados obtidos a partir da aplicação do ISP, da SUDS e do registro do aparelho de 
biofeedback, em cada sessão em que havia a exposição à RV estão distribuídos na Figura 1. Os dados de P1 estão na parte superior e de P2 na parte inferior, sendo apresentados em uma escala de 0 a 10 . Os escores do ISP foram calculados a partir da soma dos escores de cada item e, então, corrigidos para essa escala. O escore da SUDS não precisou ser corrigido. A medida registrada pelo aparelho de biofeedback foi apresentada em porcentagem de variação da resposta galvânica da pele $(\% \Delta \mu \mathrm{S})$, corrigido para a escala de 0 a 10. As linhas tracejadas na vertical após as Sessões 5 e 13 indicam, respectivamente, início e término da intervenção.

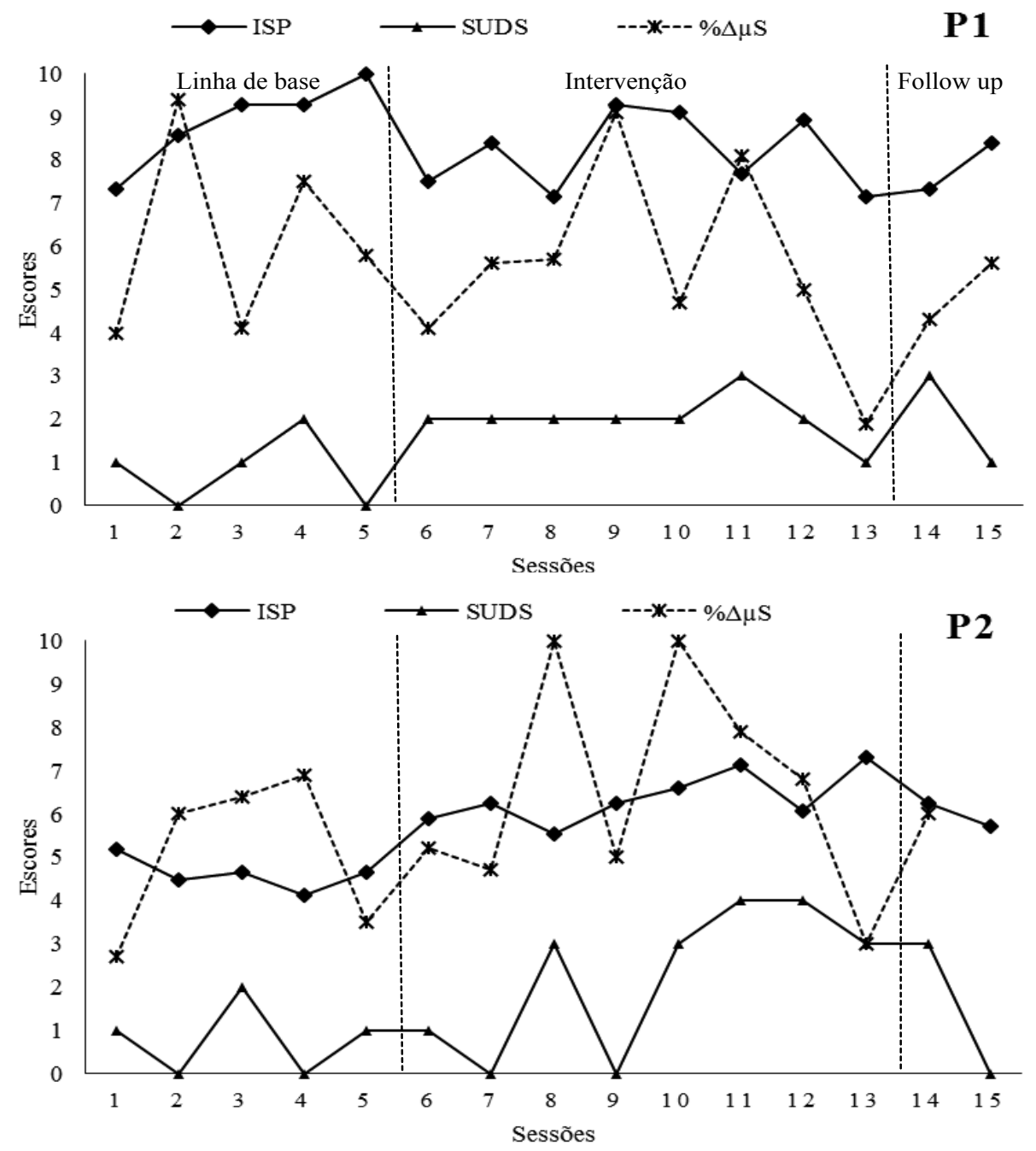

Figura 1. Distribuição dos escores corrigidos para uma escala de 0 a 10 do ISP, SUDS e porcentagens de variação da resposta galvânica da pele, em 15 sessões de exposição à $\mathrm{RV}$, de $\mathrm{P} 1$ (parte superior) e $\mathrm{P} 2$ (parte inferior). A medida da resposta galvânica da pele da última sessão de follow up do P2 não foi registrada por falha no equipamento.

Observa-se na Figura 1 que os escores obtidos pela SUDS variam de 0 a 3 para P1 e de 0 a 4 para $\mathrm{P} 2$, ao longo das 15 sessões. A SUDS era aplicada ao fim da exposição e valores próximos a zero indicam baixo nível de ansiedade sentida. P1 apresentou variação entre 0 e 3 somente nas sessões de linha de base, e após esse ponto a me- dida mínima verificada foi um (última sessão de intervenção), sendo dois o valor assinalado durante a maior parte das sessões. No caso de $\mathrm{P} 2$, a SUDS teve maior variação ( 0 a 4$)$ e mais irregularidade do que os dados de P1, variando de uma sessão para outra, na maior parte delas. Ainda na Figura 1, observa-se alta variabilidade nos dados 
da variação da resposta galvânica da pele (medida em microsiemens - $\mu S$ ), para P1 e P2, em todas as fases do estudo (linha de base, intervenção e follow up). P1 variou de 2 a 9 e $\mathrm{P} 2$ variou de 3 a 10. P2 apresentou aumento discreto após o início da fase de intervenção, havendo sessões nas quais a alteração da medida atingiu o escore 10 (Sessões 8 e 10). As sessões marcadas por elevada alteração da medida de $\mathrm{P} 2$ coincidiram com as sessões em que houve troca dos cenários (Sessões 6, 8, 10 e 12). Para P1, não houve diferença nas alterações da medida entre as fases do procedimento e as sessões marcadas por nível elevado de alterações da medida (Sessões 2, 9 e 11) não coincidiram com as trocas dos cenários.

Os dados registrados a partir da aplicação do ISP apresentaram menor variação, se comparados às outras medidas ( 7 a 10 para $\mathrm{P} 1$ e 4 a 7 para $\mathrm{P} 2$ ). Diferente de $\mathrm{P} 1$, que não apresentou variabilidade entre as fases do procedimento (variou entre 7 e 10 pontos em cada fase), no caso de P2 a variação do ISP foi diferente entre as sessões de linha de base (4 e 5) para as sessões de intervenção e follow up (6 e 7). Os dados do ISP tanto para P1 quanto para P2 apresentaram amplitude de 3 pontos na escala. Não houve aparente correspondência entre os dados do ISP com os dados da SUDS e do aparelho de biofeedback, tanto para P1 quanto para P2.

A Figura 2 apresenta os escores obtidos nos inventários SPIN, BDI e BAI, em cinco aplicações ao P1 (parte superior) e ao P2 (parte inferior). Escores acima de 19 no SPIN indicam presença de sintomas compatíveis com transtorno
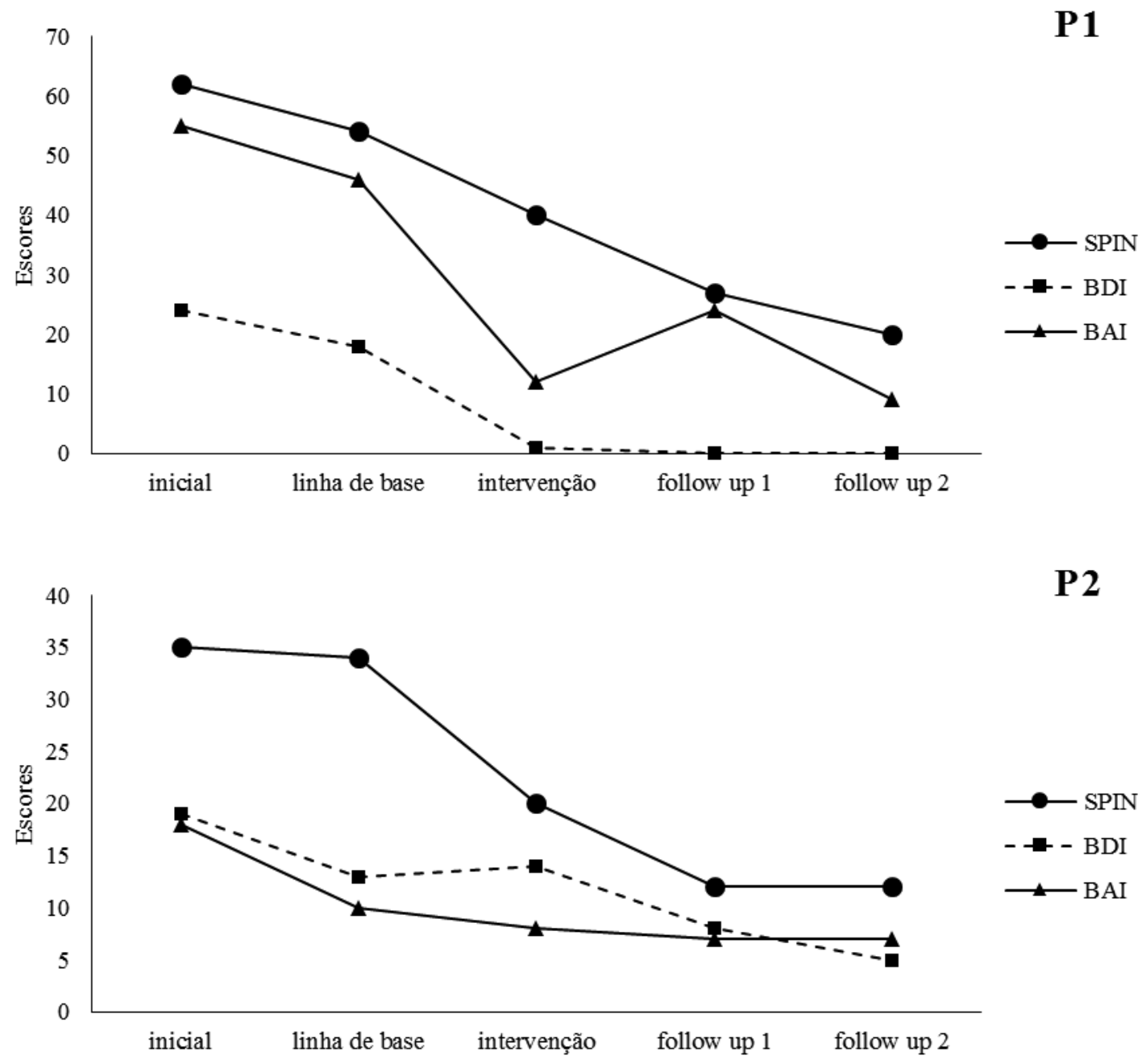

Figura 2. Escores dos testes SPIN, BDI e BAI em cinco aplicações ao P1 e ao P2. As aplicações foram feitas na sessão inicial, término da linha de base, término da intervenção, $\mathbf{1}^{\mathbf{a}}$ e $\mathbf{2}^{\mathbf{a}}$ sessões de follow up. 
de ansiedade social e escores acima de 20, para o BDI e o BAI, indicam possibilidade de depressão e ansiedade, respectivamente, em níveis moderados.

Observa-se na Figura 2 que ao longo das aplicações dos inventários SPIN, BDI e BAI os escores obtidos por de P1 e P2 diminuíram gradualmente, na maior parte das aplicações. Os escores dos três inventários decresceram na última aplicação (Follow up 2) em relação ao escore inicial nos dois casos. As reduções dos escores do SPIN, BDI e BAI da primeira aplicação (sessão inicial) para última aplicação (Follow up 2) foram de $68 \%, 100 \%$ e $84 \%$, respectivamente, para $\mathrm{P} 1$ e $66 \%, 74 \%$ e $61 \%$, respectivamente, para $\mathrm{P} 2$. Os escores obtidos por P1 e P2 na última aplicação dos inventários BDI e BAI reduziram para a faixa indicada por sintomas mínimos (Cunha, 2001). O escore do SPIN, obtido na última aplicação indicou presença de sintomas de transtorno de ansiedade social para P1 (20), apesar da proximidade ao escore mínimo (19) e reduziu para o mínimo para P2 (7).

\section{Discussão}

Os efeitos do programa de intervenção realizado, que utilizou como recurso terapêutico exposições à RV serão discutidos a fim de verificar mudanças comportamentais relacionadas à validade interna e à validade externa da intervenção. A validade interna, entendida como a mudança comportamental observada durante a intervenção, foi verificada a partir dos instrumentos de medida utilizados pré e pós-intervenção (SPIN, BDI e BAI) e a validade externa percebida como a qualidade da mudança para além do ambiente de pesquisa e intervenção (Del Prette \& Del Prette, 2008), foi verificada em relatos feitos nas sessões e nas Folhas de Registro Comportamental sobre os comportamentos apresentados pelos participantes nos contextos sociais atuais.

Os comportamentos relatados por P1 e P2 durante a sessão inicial e na linha de base podem ser compreendidos dentro da categoria diagnóstica do transtorno de ansiedade social, conforme descrito por Rocha et al. (2012) e Zamignani e
Banaco (2005), tanto pela história de aquisição quanto pela manutenção do repertório comportamental. Os participantes P1 e P2 apresentavam grande dificuldade para enfrentar e permanecer em atividades em contexto social; iniciar e manter diálogos com outras pessoas; engajar-se em interações íntimas e criar vínculos; frequentar ambientes públicos; participar de reuniões; criar laços afetivos com outras pessoas. Os contextos geralmente eram sob observação de outras pessoas, situações de desempenho de atividades em público, situações de interação verbal íntima e situações nas quais era requerido comportamento assertivo.

Quanto ao grau de familiaridade com os recursos tecnológicos, Barbosa (2013) e Zacarin et al. (2017) argumentam que esse aspecto da exposição é uma importante variável para a promoção de senso de presença sentido pelo indivíduo exposto à $\mathrm{RV}$ e níveis elevados de senso de presença são requisitos para melhores resultados ao tratamento. No presente estudo, foi aplicado o Inventário de Senso de Presença (ISP) ao fim de cada exposição e foram observados níveis elevados de presença para $\mathrm{P} 1$, nas 15 sessões de exposição, com a mesma variação ao longo do estudo. Para P2, houve discreta variação entre as fases do estudo. Em geral, P2 relatou menos presença que P1 durante todo o estudo. Entretanto, P2 relatou um aumento gradual de presença a partir do início das sessões de intervenção, atingindo níveis relatados por P1. De acordo com Barbosa (2013), ambientes que requerem maior quantidade de ações do indivíduo imerso podem produzir níveis mais elevados de presença. Fato que pode ser observado neste estudo, em que uma das mudanças decorridas da linha de base para a intervenção caracteriza-se pelo aumento de estímulos ambientais (em geral, aversivos para um indivíduo com transtorno de ansiedade social, como aglomeração de avatares em interação verbal entre si e com o participante) e aumento de recursos interativos. Na fase de intervenção, P1 e P2 alteravam significativamente o ambiente a partir da forma como interagiam com o simulador, sendo um possível indicador do aumento do senso de presença de $\mathrm{P} 2$ em comparação à linha de base. 
Conforme os resultados observados do ISP para P1 e P2, não foi possível estabelecer relação com os resultados da SUDS e do aparelho de biofeedback. Tanto P1 quanto P2 foram expostos à mesma sequência de cenários na fase de intervenção, mas a forma como os participantes responderam às solicitações da SUDS não se assemelham às mudanças da medida galvânica da pele. Esses achados sugerem que: (a) essa medida da resposta galvânica da pele pode não refletir adequadamente estados de ansiedade (verificados a partir da SUDS) ou (b) variáveis não controladas interferiram nas medidas (por exemplo, houve falta de controle da temperatura ambiental, que poderia interferir com a resposta galvânica da pele); e (c) o nível de senso de presença relatado pode não estar relacionado ao nível de ansiedade. Achados em estudos da bibliografia relativos à correlação positiva entre senso de presença e medidas de ansiedade (ver Hartanto et al., 2014; Morina et al., 2014; Powers et al., 2013; Price et al., 2011) contrariam a última hipótese citada.

Os dados relativos à medida galvânica da pele, obtidos a partir da porcentagem de variação ao longo das sessões com exposição à RV (Figura 1), indicaram que tanto $\mathrm{P} 1$ quanto $\mathrm{P} 2$ tiveram as medidas da resposta galvânica da pele alteradas durante cada sessão em no mínimo $20 \%$ ( 2 pontos na referida escala) no caso de P1 (13 sessão) e $30 \%$ (3 pontos na escala) no caso de P2 $\left(1^{\mathrm{a}}\right.$ e $13^{\mathrm{a}}$ sessões $)$. As mudanças nessas medidas indicaram que os cenários produziram alterações nos registros dos níveis fisiológicos dos participantes durante as exposições e atendem, assim, ao critério discutido por Owens e Beidel (2015) para a efetividade da intervenção. Entretanto, as alterações na resposta galvânica da pele não apresentaram padrão que pudessem ser relacionadas às trocas de cenários que, geralmente, incluíam adição de estímulos novos e potencialmente ansiogênicos, como novos avatares compondo o ambiente, interações verbais programadas e desempenho de tarefas sob observação de outros avatares.

Os resultados obtidos a partir da aplicação do SPIN, BDI e BAI (Figura 2) indicam, no entanto, mudanças expressivas ao longo do procedimento. Segundo critérios descritos por Cunha (2001), os escores apresentados por P1 e P2 na primeira aplicação do BAI (55 e 18 , respectivamente) e BDI (24 e 18 , respectivamente) indicam ansiedade grave para $\mathrm{P} 1$ e ansiedade leve para $\mathrm{P} 2$, e depressão moderada para P1 e leve para P2. Na última aplicação dos dois inventários, os escores obtidos indicam estados mínimos tanto para ansiedade quanto depressão. A diminuição dos escores na primeira aplicação para última aplicação do inventário de ansiedade foi de $84 \%$ no caso de P1 e $61 \%$ no caso de P2. Ressalta-se que os testes aplicados tinham como abrangência o espaço de tempo de uma semana. Em uma verificação dos itens contidos no BAI, observa-se que o que é tratado como sintomas de ansiedade correspondem também ao que Zamignani e Banaco (2005) descrevem como comportamentos respondentes de um repertório comportamental de ansiedade: tremores nas pernas, nervosismo, dificuldade de respirar, sudorese, palpitação, entre outros.

A partir da aplicação do SPIN também foi verificado expressiva mudança ao longo do procedimento. Entre a primeira aplicação (escore 62 para P1 e 35 para P2) e a última aplicação (escore 20 para P1 e 12 para P2) do SPIN foi observado diminuição de $68 \%$ e $62 \%$ (P1 e P2, respectivamente). De acordo com a análise descritiva da categoria diagnóstica do transtorno de ansiedade social proposta por Silvares e Meyer (2000) e da análise dos processos comportamentais envolvidos na ansiedade (Zamignani \& Banaco, 2005), os comportamentos verificados pelo SPIN podem ser analisados como comportamentos operantes de um repertório característico de ansiedade (por exemplo, evito ir a festas, evito falar com pessoas que não conheço, evito falar com qualquer autoridade, entre outros). Entretanto, ressalta-se que em uma análise funcional adequada, baseada na abordagem analítico-comportamental, outros eventos além das respostas devem ser considerados, como a ocasião na qual a resposta ocorre e as consequências do responder, além das operações estabelecedoras que determinam momentaneamente eventos como reforçadores. 
A partir dos registros obtidos com a Folha de Registro Comportamental e registros não sistemáticos dos relatos verbais durante as sessões foi possível observar mudanças em padrões comportamentais de aproximações de situações que antes eram evitadas ou suportadas com muito sofrimento. Durante a última sessão de follow up, $\mathrm{P} 2$ relatou que iniciou trabalho em uma empresa na função de operador de call-center e descreveu como um ambiente dinâmico, em postos de trabalho. Descreveu sua atribuição como receber telefonemas de clientes da operadora para solucionar problemas diversos. A partir do breve relato, observa-se característica de contexto altamente social do trabalho desempenhado. Segundo a descrição de P2, colegas de trabalho convidaram-no para um evento em happy hour e diante do convite, e apesar de ter sentido "frio na barriga" aceitou. Para P2, o evento social "foi agradável e divertido", e relatou terem comentado sobre a importância de repetirem o evento em breve. Ao início da intervenção, foi observada baixa frequência de atividades de lazer em contexto social. O episódio comportamental relatado ilustra competências sociais desenvolvidas ao longo do processo terapêutico (como assertividade, iniciar e manter conversação, manutenção de vínculo e intimidade) e dos reforços obtidos a partir da própria interação social (como aprovação social, sentimento de prazer, "bem-estar").

As competências sociais também podem ser observadas em $\mathrm{P} 1$, com generalização para o contexto natural, conforme pode ser verificado em um relato feito por P1 em uma das Folhas de Registro Comportamental. P1 relatou que em uma das vezes em que se dirigia para a Clínica Psicológica encontrou-se ao acaso com uma colega de sala de aula indo para o mesmo local. Diante da situação, P1 iniciou uma interação breve e, apesar de ter "ficado nervoso e quase com falta de ar", conseguiu manter o diálogo, relatando para a colega informações sobre o curso da própria terapia. Como consequência, P1 descreveu que ficou "orgulhoso de si" e relatou subprodutos emocionais de felicidade por ter feito o enfrentamento. A interação foi agradável para P1 e teve por consequência a aprovação social da colega e a companhia pelo trajeto caminhado, ambos reforçadores positivos para novas apresentações dos comportamentos de iniciação de conversa, manutenção de vínculo e intimidade. Tais resultados corroboram validade externa ao procedimento ao demonstrar que competências desenvolvidas com o recurso da exposição à RV tiveram generalização para fora do ambiente clinicamente controlado.

Ao longo do procedimento foram verificadas variáveis não controladas que podem ter interferido nos resultados de: (a) senso de presença, (b) resposta galvânica da pele. Sugere-se novos estudos sobre as variáveis ambientais que podem interferir sobre as medidas, como temperatura ambiental e umidade relativa do ar, questões hormonais, privação de sono e de alimento, expectativas sobre resultados. Outros aspetos também poderão ser modificados/controlados em estudos futuros, como o registro do repertório operante inicial de interação social (por exemplo, habilidades sociais), para que se possa verificar se a intervenção contribuiu para ampliar esse repertório; a utilização de outros delineamentos experimentais, como um delineamento de linha de base múltipla entre participantes, o que pode aumentar o controle de variáveis externas. Estudos desse tipo poderão contribuir para intervenções mais eficazes no futuro, importando ainda a possível análise sobre o senso de presença sentido em cada cenário, assim como o impacto de recursos interativos mais realísticos ao indivíduo, como luvas com sensibilidade tátil em relação aos estímulos virtuais. A tecnologia de RV está em pleno desenvolvimento e é característica das ciências psicológicas incorporar aos seus estudos a avaliação formal de novas tecnologias para dimensionar o impacto de seus usos como recurso terapêutico. Esses recursos podem ser alternativas às técnicas já utilizadas, como a exposição com prevenção de respostas e a dessensibilização sistemática (Barbosa, 2013; Barbosa \& Lima, 2014). Além disso, a RV pode contribuir como contexto útil para promoção da terapia verbal, aliando-se outros recursos já utilizados. 


\section{Referências}

American Psychiatric Association. (2013). Diagnostic and statistical manual of mental disorders ( $5^{\text {th }}$ ed.). Arlington, VA: American Psychiatric Publishing.

Anderson, P. L., Price, M., Edwards, S. M., Obasaju, M. A., Schmertz, S. K., Zimand, E., \& Calamaras, M. R. (2013). Virtual reality exposure therapy for social anxiety disorder: A randomized controlled trial. Journal of Consulting and Clinical Psychology, 81(5), 751-760. doi: 10.1037/ a0033559

Barbosa, J. I. C. (2013). Terapia por realidade virtual (VRET): Uma leitura analítico-comportamental. Boletim Contexto - ABPMC, 38, 113-132. Recuperado em http://abpmc.org.br/arquivos/publicacoes/1405369702866768607035.pdf

Barbosa, J. I. C., \& Lima, L. S. (2014). Terapia de exposição ao estímulo fóbico com o uso de Realidade Virtual: Uma revisão bibliográfica. In $\mathrm{N}$. B. Borges, L. F. G. Aureliano, \& J. L. Leonardi (Eds.), Comportamento em Foco (Vol. 4, pp. 73 82). São Paulo, SP: ABPMC. Recuperado em http://abpmc.org.br/arquivos/publicacoes/1 41622281567a933aae65d.pdf

Beidel, D. C., Alfano, C. A., Kofler, M. J., Rao, P. A., Scharfstein, L., \& Wong Sarver, N. (2014). The impact of social skills training for social anxiety disorder: A randomized controlled trial. Journal of Anxiety Disorders, 28, 908-918. doi: 10.1016/j.janxdis.2014.1009.1016

Cunha, J. A. (2001). Manual da versão em português das Escalas Beck. São Paulo, SP: Casa do Psicólogo.

Del Prette, Z. A. P., \& Del Prette, A. (2008). Significância clínica e mudança confiável na avaliação de intervenções psicológicas. Psicologia: Teoria e Pesquisa, 24(4), 497-505. doi: 10.1590/ S0102-37722008000400013

Harris, S. R., Kemmerling, R. L., \& North, M. M. (2002). Brief virtual reality therapy for public speaking anxiety. Cyberpsychology \& Behavior, 5(6), 543-550. doi: $10.1089 / 109493102321018187$

Hartanto, D., Kampman, I. L., Morina, N., Emmelkamp, P. G. M., Neerincx, M. A., \& Brinkman, W.-P. (2014). Controlling social stress in virtual reality environments. PLOS ONE 9(3). e92804. doi: 10.1371/journal.pone.0092804
Heijden, N. T., \& Brinkman, W. P. (2011). Design and evaluation of a virtual reality exposure therapy system with automatic free speech interaction. Journal of CyberTherapy \& Rehabilitation, 4(1), 41-56. Retrieved from http://repository. tudelft.nl/islandora/object/uuid:924c076e-5bf3$4 \mathrm{c} 45-\mathrm{b} 714-94 \mathrm{~d} 391 \mathrm{cf} 9020$ ?collection $=$ research

Holt, C. S., Heimberg, R. G., Hope, D. A., \& Liebowitz, M. R. (1992). Situational domains of social phobia. Journal of Anxiety Disorders, 6(1), 6377. doi: 10.1016/0887-6185(92)90027-5

Kampmann, I. L., Emmelkamp, P. M., Hartanto, D., Brinkman, W. P., Zijlstra, B. J., \& Morina, N. (2016). Exposure to virtual social interactions in the treatment of social anxiety disorder: A randomized controlled trial. Behaviour Research and Therapy, 77, 147-56. doi: 10.1016/j. brat.2015.1012.1016

Klinger, E., Bouchard, S., Legeron, P., Roy, S., Lauer, F., Chemin, I., \& Nugues, P. (2005). Virtual reality therapy versus cognitive behavior therapy for social phobia: A preliminary controlled study. Cyberpsychology \& Behavior, 8(1), 7688. doi: $10.1089 / \mathrm{cpb} .2005 .8 .76$

Leonardi, J. L., Borges, N. B., \& Cassas, F. A. (2012). Avaliação funcional como ferramenta norteadora da prática clínica. In N. B. Borges \& F. A. Cassas (Eds.), Clínica analítico-comportamental: Aspectos teóricos e práticos (pp. 105-109). Porto Alegre, RS: Artmed.

Morina, N., Brinkman, W-P., Hartanto, D., \& Emmelkamp, P. M. G. (2014). Sense of presence and anxiety during virtual social interactions between a human and virtual humans. PeerJ 2:e337. doi: 10.7717/peerj.337

Nobile, G. F. G., Garcia, V. A., \& Bolsoni-Silva, A. T. (2017). Análise sequencial dos comportamentos do terapeuta em psicoterapia com universitários com transtorno de ansiedade social. Perspectivas em Análise do Comportamento, 8, 16-31. doi: 10.18761/pac.2016.030

Osório, F. L., Crippa, J. A. S., \& Loureiro, S. R. (2009). Validação transcultural da versão para o português do Brasil do Social Phobia Inventory (SPIN): Estudo dos itens e da consistência interna. Revista Brasileira de Psiquiatria, 31(1), 2529. doi: $10.1590 / \mathrm{S} 1516-44462008005000018$

Owens, M. E., \& Beidel, D. C. (2015). Can virtual reality effectively elicit distress associated with social anxiety disorder? Journal of Psychopa- 
thology and Behavioral Assessment. 37, 296305. doi: 10.1007/s10862-014-9454-X

Powers, M. B., Briceno, N. F., Gresham, R., Jouriles, E. N., Emmelkamp, P. M. G., \& Smits, J. A. J. (2013). Do conversations with virtual avatars increase feelings of social anxiety? Journal of Anxiety Disorders, 27(4), 398-403. doi: 10.1016/j.janxdis.2013.03.003

Price, M., Mehta, N., Tone, E. B., \& Anderson, P. L. (2011). Does engagement with exposure yield better outcomes? Components of presence as a predictor of treatment response for virtual reality exposure therapy for social phobia. Journal of Anxiety Disorders, 25(6), 763-770. doi: 10.1016/j.janxdis.2011.03.004

Qu, C., Brinkman, W., Ling, Y., Wiggers, P., \& Heynderickx, I. (2014). Conversation with a virtual human: Synthetic emotions and human responses. Computers in Human Behavior, 34, 58-68. doi: 10.1016/j.chb.2014.01.033

Rocha, J. F., Bolsoni-Silva, A. T., \& Verdu, A. C. M. A. (2012). O uso do treino de habilidades sociais em pessoas com fobia social na terapia comportamental. Perspectivas em Análise do Comportamento, 3(1), 38-56.

Roy, S., Klinger, E., Légeron, P., Lauer, F., Chemin, I., \& Nugues, P. (2003). Definition of a VRBased Protocol to treat social phobia. Cyberpsychology \& Behavior, 6(4), 411-420. doi: 10.1089/109493103322278808

Sampaio, N. S. de P., \& Bueno, G. N. (2011). Manejo da fobia à exposição oral: Estratégias comportamentais como agentes de controle. In C. V. B. B. Pessôa, C. E. Costa, \& M. F. Benvenuti (Eds.), Comportamento em Foco (Vol. 1, pp. 583-598). São Paulo, SP: ABPMC.

Silvares, E. F. D. M., \& Meyer, S. B. (2000). Análise funcional da fobia social em uma concepção behaviorista radical. Revista de Psiquiatria Clínica, 27(6), 329-334.

Slater, M., Pertaub, D. P., Barker, C., \& Clark, D. M. (2006). An experimental study on fear of public speaking using a virtual environment.
Cyberpsychology \& Behavior, 9(5), 627-633. doi: $10.1089 / \mathrm{cpb} .2006 .9 .627$

Stevens, S., Peters, A., Abraham, A., \& Hermann, C. (2014). Enhanced avoidance behavior in social anxiety: Evidence from a probabilistic learning task. Journal of Behavior Therapy and Experimental Psychiatry, 45, 39-45. doi: 10.1016/j. jbtep.2013.1007.1007

Sturmey, P. (1996). Functional analysis in clinical psychology. Chichester, UK: John Wiley \& Sons.

Wallach, H. S., Safir, M. P., \& Bar-Zvi, M. (2011). Virtual reality exposure versus cognitive restructuring for treatment of public speaking anxiety: A pilot study. Israel Journal of Psychiatry \& Related Sciences 48(2), 91-97. doi: 22120443

Wiederhold, B. K., Jang, D. P., Kim, S. I., \& Wiederhold, M. D. (2002). Physiological monitoring as an objective tool in virtual reality therapy. Cyberpsychology \& Behavior, 5(1), 77-82. doi: 10.1089/109493102753685908

Wolpe, J. (1978). Prática da terapia comportamental (2. ed., W. G. Clark Jr., Trad.). São Paulo, SP: Brasiliense. (Original publicado em 1973)

Zacarin, M. R. J., Borloti, E., Santos, A., Perandré, Y. H. T., Melo, C. M. \& Haydu, V. B. (2017). Senso de presença: Proposta de uma definição analítico-comportamental. Acta Comportamentalia. 25(2), 249-263.

Zamignani, D. R., \& Banaco, R. A. (2005). Um panorama analítico-comportamental sobre os transtornos de ansiedade. Revista Brasileira de Terapia Comportamental e Cognitiva, 7, 77-92. Recuperado em http://www.usp.br/rbtcc/index.php/RBTCC/article/view/44/33

Recebido: 25/10/2016

$1^{a}$ revisão: $29 / 04 / 2017$

$2^{a}$ revisão: $23 / 05 / 2017$ Aceite final: $23 / 05 / 2017$

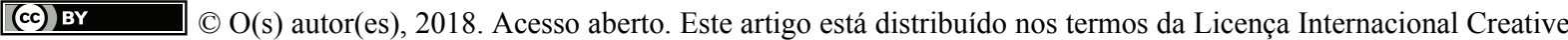
Commons Atribuição 4.0 (http://creativecommons.org/licenses/by/4.0/), que permite o uso, distribuição e reprodução sem restrições em qualquer meio, desde que você dê crédito apropriado ao(s) autor(es) original(ais) e à fonte, fornecer um link para a licença Creative Commons e indicar se as alterações foram feitas. 